\section{QUESTION 3}

If at all possible, the diagnosis should be confirmed histologically by tissue biopsy of skin or kidney to reveal the pathognomonic needle-shaped clefts in arterioles. Once established, invasive procedures known to precipitate CCE should be avoided. Anticoagulation and thrombolysis are also contraindicated. Associated risk factors such as hypertension, hypercholesterolaemia and smoking should be managed aggressively. Renal replacement therapy may be required.

Once pre-mortem diagnosis is made (less than $40 \%$ of cases) prognosis is poor. Mortality is high, with death most commonly due to ischaemic heart disease, cerebrovascular disease and renal failure, while $10.8 \%$ of patients die of the multiple cholesterol emboli syndrome.'

Heightened awareness of CCE will lead to early diagnosis, thus avoiding precipitating factors. Unnecessary immunosuppressant therapy will also be avoided since CCE can mimic the clinical presentation of systemic vasculitis. ${ }^{5}$

Our patient did not undergo angiography, nor tissue biopsy and his condition deteriorated rapidly despite stopping warfarin. There was progressive renal failure and a step-wise

1 Moolenar W, Lamers CBHW. Cholesterol crystal embolism in the Netherlands. Arch Intern Med 1996;156:653-7.

Mayo RR, Swartz RD. Redefining the incidence of clinically detectable atheroembolism. Am $₹$ Med 1996;100:524-9.

3 Falanga F, Fine MJ, Kapoor WN. The cutaneous manifesta3 Falanga F, Fine MJ, Kapoor WN. The cutaneous manifesta-
tions of cholesterol crystal embolism. Arch Dermatol 1986; 122:1194-8.

\section{Learning points}

- clinicians should have a heightened awareness of CCE

- CCE may mimic other systemic diseases eg vasculitis

- once diagnosed, precipitating factors should be avoided

Box 5

decline in cognitive function secondary to vascular dementia (brain computed tomography scan revealed multiple small ischaemic areas). He died four months after presentation. The presence of more than four risk factors together with the clinical features makes the diagnosis of CCE very likely.

\section{Final diagnosis}

Cholesterol crystal embolism.

Keywords: cholesterol crystal embolism; purple toes; renal failure; anticoagulation

\footnotetext{
4 Robson MG, Scoble JE. Atheroembolic disease. $\mathrm{Br} \mathcal{F}$ Hosp Med 1996;55:648-52.

5 Peat DS, Mathieson PW. Cholesterol emboli may mimic
} systemic vasculitis. BMF 1996;313:546-7.
Department of Surgery, Gwynedd

Hospital, Bangor, Gwynedd LL57 2PW, UK

D S Bhandarkar

T R Raju

L R Jenkinson

Correspondence to $\mathrm{Mr} \mathrm{L} \mathrm{R}$ Jenkinson

Accepted 28 May 1997

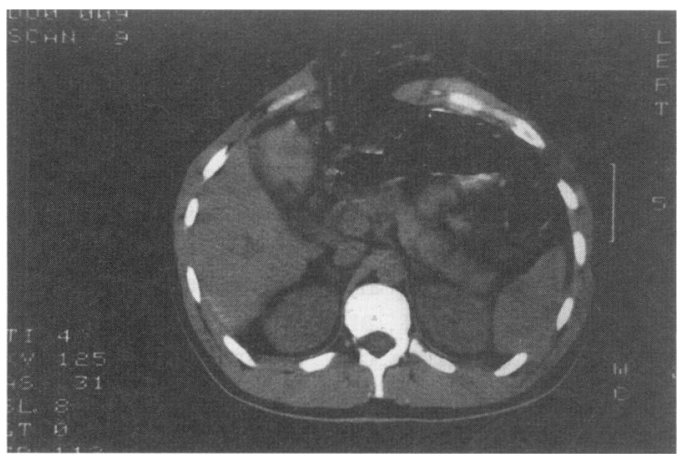

\section{Questions}

1 What is the diagnosis?

2 What is the differential diagnosis of an abdominal wall swelling appearing after blunt trauma? 


\section{Answers}

QUESTION 1

The CT scan shows the recti to be widely separated with prolapse of loops of small bowel through the ensuing defect. This confirms the swelling to be a post-traumatic abdominal wall hernia.

QUESTION 2

Differential diagnosis of an abdominal wall swelling occurring after blunt abdominal trauma includes a pre-existing hernia and a rectus sheath haematoma. It is particularly important not to confuse a hernia with the latter as a misdiagnosis may result in the swelling being incised. A lateral X-ray or a contrast series may demonstrate loops of bowel outside the abdominal cavity. Ultrasonography might be expected to differentiate between a haematoma and gas-filled bowel but CT is sometimes necessary to confirm the diagnosis.

\section{Clinical course}

At laparotomy undertaken through an upper midline incision, a $10 \mathrm{~cm}$ defect with irregular, rushed edges was noted in the supra-umbilical linea alba. A liver laceration identified on the pre-operative CT scanning had stopped bleeding and was left undisturbed. A thorough exploration excluded other visceral injuries. The edges of the hernial defect were debrided and the incision was closed with loop nylon. The patient made an uneventful recovery and was discharged home on the tenth day. He was re-admitted twice, once for aspiration of a wound seroma and subsequently with complaints of upper abdominal pain. Ultrasonography performed during the second admission revealed a haematoma under the right lobe of the liver that was found to resolve on subsequent scanning. At follow-up a year later he remains well and there is no recurrence of the hernia.

\section{Discussion}

Despite the large number of abdominal injuries occurring every year, traumatic abdominal wall hernia remains a rare occurrence. These hernias can occur as a result of a variety of injuries including impalement against handlebars, ${ }^{1}$ goring by animals, ${ }^{2}$ falls, ${ }^{3}$ seatbelt injuries, ${ }^{4}$ and road traffic accidents. ${ }^{5}$ The criteria necessary for establishing the diagnosis of a traumatic hernia are: the immediate appearance of the hernia after trauma which should not have lead to penetration of the skin, the presence of signs of the trauma at the initial medical consultation (so as to exclude a pre-existing defect), ${ }^{1}$ and the absence of an identifiable hernial sac in the defect at operation corroborating the short duration of the hernia. ${ }^{5}$

The commonest cause of a traumatic hernia seems to be a blow to the abdominal wall with a blunt object of moderate size, not small enough to penetrate the skin nor large enough

\section{Criteria for diagnosis of} post-traumatic abdominal wall hernia

- immediate appearance of the hernia after trauma that should not have resulted in penetration of skin

- presence of signs of the trauma at initial medical consultation

- absence of an identifiable hernial sac in the defect at operation

Box 1

\section{Post-traumatic abdominal wall hernias}

rare but can occur at various sites following blunt trauma

- high index of suspicion necessary for early diagnosis

- differentiation from a post-traumatic haematoma important

- associated intra-abdominal injuries common and must be excluded

- early repair of the hernia is the preferred method of treatment

Box 2

for the forces to be dissipated widely. It can also occur as a result of more widespread blunt injury. Our patient exemplifies the latter event in which the mechanism responsible appears to be a combination of local trauma and sudden rise in the intra-abdominal pressure caused by sudden contraction of the abdominal muscles. The hernias can occur through a defect in a muscle, a defect in its tendinous insertion or fascia, or as a result of avulsion of a muscle or a tendon from bone. Hernias occurring at other sites, viz, right upper quadrant, ${ }^{6}$ flank, ${ }^{5}$ and inguinal regions ${ }^{1}$ have also been described.

As with any uncommon medical problems, awareness of the entity is necessary to diagnose a traumatic abdominal hernia. The presence of local pain, bruising and a reducible swelling with a cough impulse suggest the diagnosis. Auscultation over the swelling may reveal bowel sounds. Further imaging may be necessary in case of a diagnostic difficulty.

As a traumatic hernia is often associated with intra-abdominal injuries, in most instances it should be operated upon when recognised. Even in the case of a laterally placed hernia the operation is best carried out through a midline incision as it allows for a thorough abdominal exploration. Early repair of severely traumatised tissues has been cited as a factor responsible for recurrence of traumatic hernia. ${ }^{4}$ Nonetheless, it is generally preferred over a late repair as the latter makes the hernia prone to complications such as obstruction or strangulation, requires a second hospital admission and usually necessitates use of prosthetic material to close the defect. However, sometimes in polytraumatised patients, if intra-abdominal injuries have been excluded, the repair may be 
delayed to allow stabilisation of general condition. It should be stressed that whatever the timing, adequate debridement of devitalised tissue, tension-free approximation and use of nonabsorbable material are principles paramount to the success of the repair.

1 Clain A. Traumatic hernia. Br $\mathcal{F}$ Surg 1964;51:549-50. 2 Atiemo EA, Goswami G. Traumatic ventral hernia. Trauma 1974;14:181-2.

3 Dajee $H$, Nicholson DM. Traumatic abdominal hernia. Trauma 1979;19:710-11.

Trauma 1979;19:710-11.
Payne DD, Resnicoff SA, States JD, Williams JS. Seat bel abdominal wall muscular avulsion. $\mathcal{f}$ Trauma 1973;13:262-7.

\section{Final diagnosis}

Post-traumatic abdominal wall hernia.

Keywords: hernia; abdominal trauma

5 Malangoni $M$, Condon RE. Traumatic abdominal wall hernia. F Trauma 1983;23:356-7.

6 Dubois PM, Freeman 1B. Traumatic abdominal wall hernia. f Trauma 1981;21:72-4

\section{A case of a yellow patient}

\section{MMU Chowdhury, S Natarajan}

A 60-year-old woman was referred by her general practitioner with joint pains, muscle aches and her skin becoming yellow on her trunk and limbs over a two-month period. On examination, she had the features shown in her palms (figure 1), elbows (figure 2), and shoulders (figure 3).

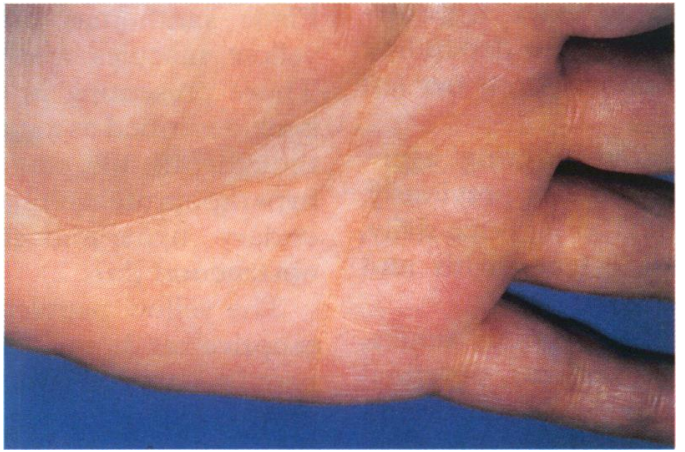

Figure 1

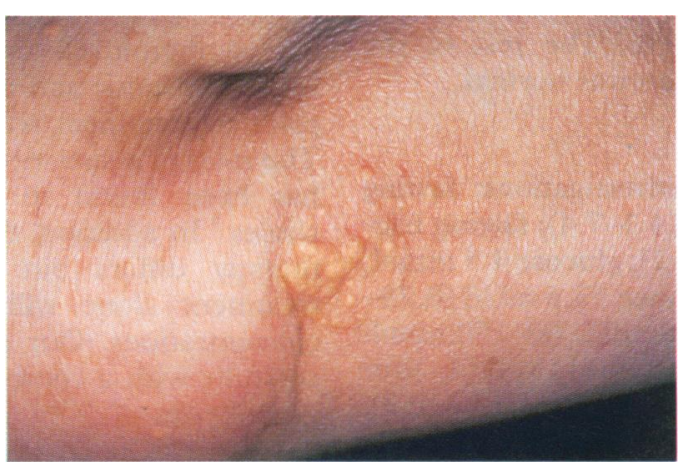

Figure 2

\section{Questions}

1 What are the abnormalities seen in the figures?

2 What further history and examination would be required?

3 What investigations would you perform?

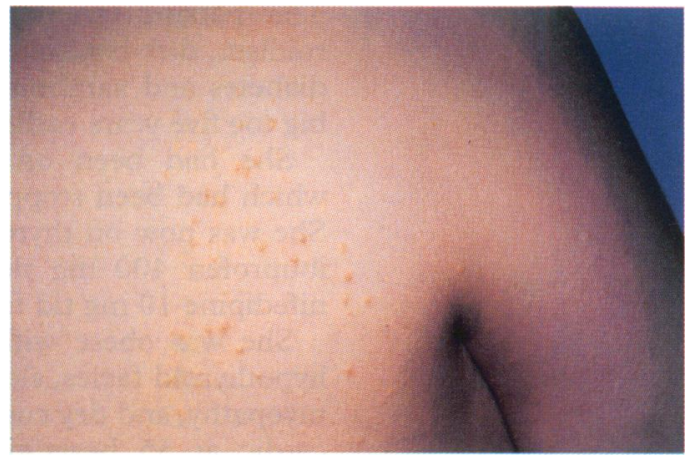

Figure 3 